\title{
Neurolinguistic analysis of the language abilities of a patient with a "double disconnection syndrome": a case of subangular alexia in the presence of mixed transcortical aphasia
}

\author{
FRA NCIS J P I R OZZOLO, KATHR Y L KERR, \\ JOH N E OBZUT, GERALDK MORLEY, JAMES V HAXBY, \\ A N D S A N R A L U D G R N \\ From the Minneapolis VA Medical Center, Minnesota, USA
}

SUMMARY In contrast to the classic form of alexia without agraphia, subangular alexia results from a single lesion located deep in the white matter of the left parietal lobe. In the present report, a patient with subangular alexia and features of mixed transcortical aphasia is described. Neurolinguistic findings include: alexia without agraphia, paucity of spontaneous speech, moderate auditory comprehension difficulty, excellent repetition, echopraxia, colour agnosia, and naming disorder. Neurolinguistic tests revealed intact phonological organisation and "grammatical filter." Our studies revealed a "double disconnection syndrome," the co-existence of two relatively rare neurobehavioural disorders. Furthermore, the studies reported here clearly show dissociations of language functions in both the visual and auditory modality, which demonstrates that the stages of language processing are separable.

The classic syndrome of alexia without agraphia usually results from two lesions-one involving the left occipital lobe and one involving the splenium of the corpus callosum. Dejerine ${ }^{1}$ described the first post mortem findings of a patient with this form of reading disability. He reasoned that the occipital infarct caused destruction of the primary visual projection zone of the left hemisphere and that the callosal lesion prevented visual information processed in the right calcarine cortex from being transmitted to the "centre of optic images" in the angular gyrus. Since the angular gyrus was spared, spontaneous writing and writing to dictation were intact.

One particularly intriguing variant of alexia without agraphia is the subangular alexia syndrome. $^{23}$ In contrast to the classic form of alexia without agraphia, subangular alexia results from

Address for reprint requests: Dr Francis J Pirozzolo, GRECC-127F, Minneapolis VA Medical Center, Minneapolis, Minnesota 55435, USA.

Accepted 30 July 1980 a single white matter lesion located deep in the left parietal lobe which disconnects the angular gyrus from both visual cortices. The associated clinical findings can include aphasia or sensory symptomatology, but not agraphia. Since the lesion does not involve the calcarine cortex, intact visual fields are often found in cases of subangular alexia. The patient to be described in the present report manifested the neuropsychological signs of subangular alexia (reading disability, colour agnosia, intact spontaneous writing and writing to dictation and preserved visual fields) and, in addition, had features of mixed transcortical aphasia (paucity of spontaneous speech, moderate semantic-dissociative auditory comprehension difficulty, and excellent repetition).

\section{Case report}

The patient was a 63 year old man who presented to the Minneapolis VA Medical Center with aphasia due to a left parietal haemorrhage. His previous medical 
history included a brainstem ischaemic episode six months prior to this admission. He was treated with coumadin and there were no residual neurological difficulties. Neurological examination on admission showed right hemiparesis, decreased speech output, word finding difficulty, and moderate auditory comprehension difficulty. He was unable to read but could write acceptably. The patient was followed and after six weeks his language behaviour had stabilised. CT scan at that time showed a large white matter lesion underlying the angular gyrus (see figure). In addition, a poorly visualised second lesion enchroaching on the thalamus was suspected. He had a mixed transcortical aphasia, shown by intact repetition, grammatically correct syntax, absence of inhibition, and moderate auditory comprehension difficulties. His reading was severely disturbed, with writing only mildly affected. The patient was echopraxic and this disinhibition occurred even when the examiner attempted to resist the patient's mimicry of gestures. He could write his name, address and telephone number without error. Writing to dictation was essentially normal for single, high frequency words and for short sentences. Spontaneous writing was also relatively intact and judged to be somewhat better than his auditory comprehension.
Although the patient's writing was only mildly impaired, he could not read even simple, monosyllabic words. This alexia affected both oral reading (decoding) and silent reading comprehension. On the aphasia tests, the subject was unable to match written words with common objects whose names were written on cards. In addition, the patient could not execute the whole body commands that were printed on cards. Colour naming, colour matching, and object naming were also moderately impaired.

\section{Neurolinguistic studies}

Since the predominant neurolinguistic feature of the transcortical aphasias is an intact ability to repeat what the subject has heard, we studied our patient's language ability through tests of repetition. We found that the use of a nonterminal intonation contour facilitated the subject's response, thus supporting the idea that disinhibition and completion phenomena are responsible, in part, for well-preserved repetition in transcortical aphasia. Most studies of the repetition behaviour of transcortical aphasics have used the term "echolalia" to refer to this phenomenon, but recent investigations ${ }^{4-6}$ have shown that the repetition uttered by these aphasics is not totally automatic, exact, or parrot-like. Repetition is influenced by the patient's

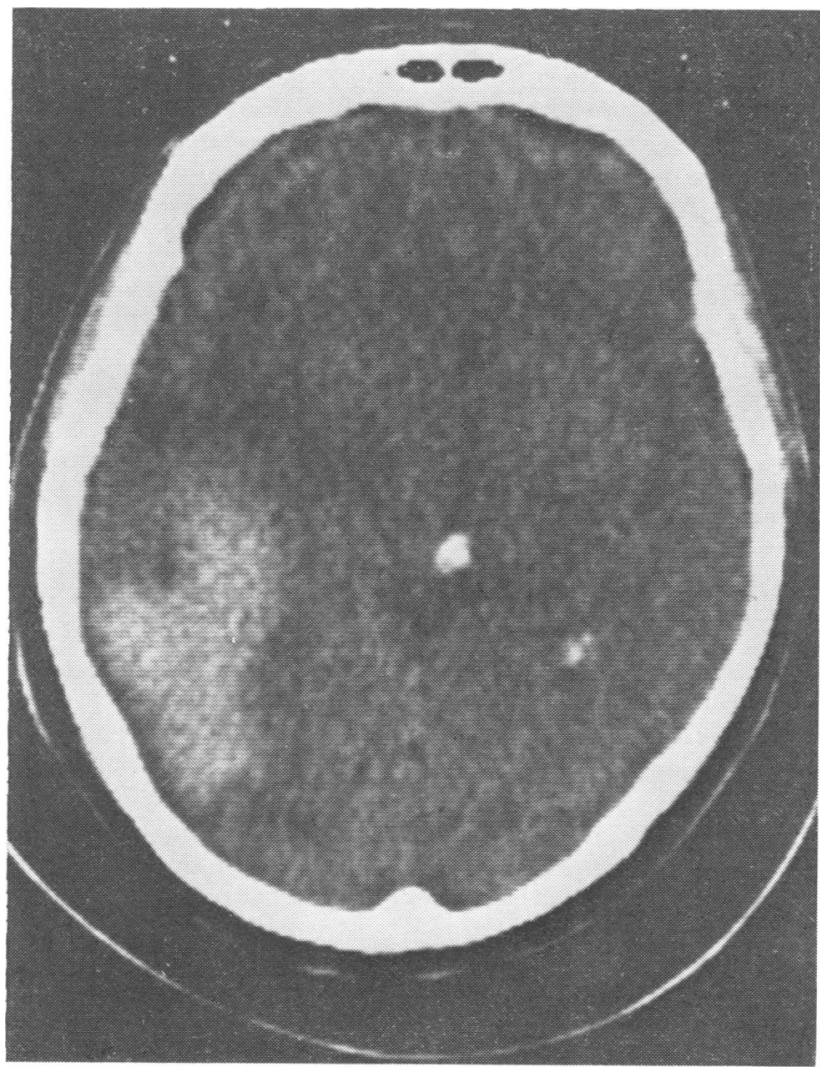

Figure CT scan showing lesion in left angular gyrus and possibly a second lesion encroaching on right thalamus. 
preserved linguistic competence. In the following section, the neurolinguistic tests that were administered to our patient will be described. The results of this evaluation clearly show the linguistic dissociations that can be distinguished through an analysis of the subject's information processing systems.

\section{Phonological organisation}

The patient did not show difficulties in producing the correct suprasegmental patterns of English language. He did not have articulatory, prosodic or intonational disturbances. When presented with noun compounds with incorrect stress or intonation patterns (for example, "My mother was ba king'a cake), our subject corrected the phonological deviations in his repetition. Similarly, when the examiner named objects presented to the subject using minor phonemic errors, he also corrected the word in his response (for example, "This is a sook" (book). When difficult articulatory sequences were produced (for example, Italian Communist Insurrection) our patient reproduced these words correctly. To illustrate that his repetition was not a parrot-like echoing of verbal stimuli, German and Italian words and sentences were used, such as the following:

E: Meine Mutter will ans Meer fahren.

S: (silent).

E: Il viaggio da Minneapolis a St. Paul é non lungo.

S: Minneapolis-St. Paul.

The second example illustrates the subject's tracking of the verbal stimuli and repetition only when the segments aroused familiar phonological associations.

\section{Syntactic organisation}

Several tests of grammatical function were administered in order to examine a deeper level of neurolinguistic performance. Sentences containing number disagreement (for example, "The pen and pencil is in my pocket") or syntactic anomalies (for example, "Hand the paper to she") were spontaneously corrected by our subject in his response. Conversely, sentences with semantic anomalies (for example, "Elephants are smaller than ants") were repeated without alteration. These results strongly suggest that this aphasic's repetition is constrained by a "grammatical filter". Stylistically altered sentences that contained syntactic violations were corrected by our subject. Sentences containing factual or semantic deviations were repeated verbatim. While normal subjects perform similarly on the latter tasks, it is generally believed that comprehension of the anomaly delays response. ${ }^{6}$ Our subject was spontaneous in his repetition and apparently unaware of the semantic or factual anomalies presented to him.

\section{Discussion}

The neurolinguistic performance of our patient is particularly interesting in that it illustrates dissociations between the more automatic features of linguistic processing in both oral and written language. In oral language, lower level processes such as auditory discrimination, phonological skills and syntactic organisation are well preserved and unaffected by lesions outside the classical language zones. The semantic representation of these phonemic and syntactic features is disrupted by the lesion which disconnects the auditory association cortex from the cortical regions thought to store the semantic representations of the phonemic patterns. This dissociation, or disconnection as it has been called by Geschwind, ${ }^{7}$ has been found by other researchers. ${ }^{48}$ The study of this case supports the now abundant evidence that syntactic and semantic components of language are separable and can be selectively impaired by brain damage.

A second disconnection, one involving written language, can also be seen in the neurolinguistic performance of our subject. The patient had intact lower level visual processes including visual acuity and discrimination. Perhaps only through the sparing of phonological ability, and a phonemeto-grapheme translation strategy, can spontaneous writing and writing to dictation be preserved. $\mathrm{He}$ could reproduce in writing the verbal symbols that he could not name or read. This disconnection, anatomically between the posterior cerebral areas that subserve visual information processing and the area that subserves the lexical process, impaired our subject from appreciating the meaning of orthographic symbols. As in the case of oral language, our patient could successfully carry out the more automatic levels of processing but this information was not coded successfully into meaning.

The authors acknowledge with thanks the assistance of Dr Alan Rubens, who provided several valuable observations of our patient, and Drs Samuel Greenblatt, Robert Brookshire, and Ola Selnes whose critical comments were helpful in preparing this manuscript. This research was supported, in part, by grants to the first author from the Veterans Administration and from the Minnesota Medical Foundation.

\section{References}

1 Dejerine $\mathrm{H}$. Contribution a l'étude anatomopathologique et clinique des differentes varietes de cecite verbale. Memoires de la Societe de Biologie 1891, 4:61-90.

2 Greenblatt S. Alexia without agraphia or hemianopsia. Brain 1973; 96:307-16.

3 Greenblatt, S. Subangular alexia without agraphia or hemianopsia. Brain Lang 1976; 3:229-45. 
4 Whitaker $\mathrm{H}$. A case of isolation of the language function. In: Whitaker $\mathrm{H}$ and Whitaker $\mathrm{HA}$ eds. Studies in neurolinguistics. New York: Academic Press, 1976.

5 Rubens AB. Transcortical motor aphasia. In: Whitaker $\mathrm{H}$ and Whitaker HA eds. Studies in neurolinguistics. New York: Academic Press, 1976.

6 Davis L, Foldi N, Gardner H, Zurif E. Repetition in the transcortical aphasias. Brain Lang 1978; 6: 226-38.

7 Geschwind N. Disconnexion syndromes in animals and man. Brain 1965; 88:237-94.

8 Zurif E, Caramazza A. Psycholinguistic structures in aphasia: Studies in syntax and semantics. In: Whitaker $\mathrm{H}$ and Whitaker HA eds. Studies in Neurolinguistics. New York: Academic Press, 1976. 